\title{
Edge Semientire Cutvertex Graph
}

\author{
Venkanagouda M. Goudar \\ Sri Gouthama Research centre,(Affiliated to Kuvempu University), Department of \\ Mathematics, Sri Siddhartha Institute of Technology, \\ Tumkur, Karnataka- 572105 \\ Email:vmgouda@gmail.com
}

\begin{abstract}
In this communications, the concept of the edge semientire cutvertex graph is introduced. We present characterization of graphs whose edge semientire cutvertex graph is planar, nonouterplanar. Further, Also we establish a characterization of graphs whose edge semientire cutvertex graphs are eularian, hamiltonian and the graphs whose crossing number one.
\end{abstract}

Keywords: crossing number, cutvertex graph, edge semientire graph, inner vertex number, line graph, outerplanar.

\section{Introduction}

The concept of lict graph $n(G)$ of a graph $\mathrm{G}$ was introduced by Kulli and $\mathrm{M} \mathrm{H}$ Muddebihal [3], is the graph whose vertex set is the union of the set of edges and the set of cutvertices of $G$ in which two vertices are adjacent if and only if the corresponding edges of $\mathrm{G}$ are adjacent or the corresponding members of $\mathrm{G}$ are incident. A new concept of a graph valued functions called the edge semientire graph $e_{e}(G)$ was introduced by Kulli [4]. For the planar graph $G$, the edge semientire graph is the graph whose vertices can be put in one to one correspondence with the edges and regions of $G$ in such a way that two vertices of $e_{e}(G)$ are adjacent if and only if the corresponding elements of $G$ are adjacent. The line graph $L(G)$ of a graph $G$ is the graph whose vertex set coincides with the edge set of $G$ and in which two vertices are adjacent if and only if the corresponding edges are adjacent in G. A regionvertex is a vertex in edge semientire graph corresponding to the region of $\mathrm{G}$. 
The edge semientire cutvertex graph of a planar graph $G$ denoted by $e_{c}(G)$ and is defined as the graph whose vertex set is the union of the set of edges, set of cutvertices and set of regions of $\mathrm{G}$ in which two vertices are adjacent if and only if the corresponding edges are adjacent, edges are incident to the cutvertex, edges are lies on the region and cutvertices are adjacent in $\mathrm{G}$.

The edgedegree of an edge is defined in [4]. The inner vertex number $i(G)$ of a graph $\mathrm{G}$ is the minimum number of vertices not belonging to the boundary of the exterior region in any embedding of $G$ in the plane. A graph $G$ is said to be minimally nonouterplanar if $i[G]=1$, as was given by Kulli [5]. The regionvertex is a vertex obtained from the region of $\mathrm{G}$ and the vertex which has finite region is called inner regionvertex.

All the undefined terms may be referred to Harary ${ }^{2}$. All graphs considered here are finite, undirected and without loops or multiple edges and a graph $\mathrm{G}$ contains at least two cutvertices.

\section{Preliminaries}

We need the following Theorems for the proof of our further results.

Theorem 2.1: If $G$ is a $(p, q)$ graph whose vertices have degree $d_{i}$ then $L(G)$ has $q$ vertices and $q_{L}$ edges where $q_{L}=-q+1 / 2 \sum d_{i}^{2}$.

Theorem 2.2: The line graph $L(G)$ of a graph $G$ has crossing number one if and only if $G$ is planar and 1 or 2 holds:

1. The maximum degree $\Delta(G)$ is 4 and there is unique non cutvertex of degree.

2. The maximum degree $\Delta(G)$ is 5 , every vertex of degree 4 is a cutvertex, there is a unique vertex of degree 5 and has atmost 3 edges in any block.

Theorem 2.3: A connected graph $G$ is isomorphic to its line graph if and only if it is a cycle.

Theorem 2.4: The lict graph $n(G)$ is planar if and only if deg $v \leq 3$.

Theorem 2.5: The edge semi entire graph $e_{e}(G)$ is planar if and only if

$1 \quad G$ is a tree

2. $\quad d e g v \leq 3$ for every vertex $v$ of $G$. 
Theorem 2.6: Let $G$ be a plane graph. A necessary and sufficient condition for $e_{e}(G)$ to be eulerian is that each of the following holds:

1. Each edge of $G$ is adjacent to even number of elements

2. Each region of $G$ has even number of elements adjacent to it.

Theorem 2.7: If $G$ is a hamiltonian plane graph, then $e_{e}(G)$ is also Hamiltonian

\section{Edge Semientire Cutvertex Graph ec(G) of a Planar Graph G}

We start with a few preliminary results.

Remark 3.1: For any graph $G, L(G) \subset n(G)$ and $e_{e}(G) \subset e_{c}(G)$.

Remark 3.2: For any graph $G, e_{c}(G)$ is nonseparable.

In the following Theorem, we obtain the number of vertices and edges in edge semientire cutvertex graph.

Theorem 3.1: For any $(p, q)$ graph $G$ whose vertices have degree $d_{i}$, cutvertices $\left|c_{i}\right|=c$, regions $r, l_{i}$ be the number of edges to which cutvertex $c_{i}$ belongs and $e_{k}$ be the number of edges in which the region $r_{k}$ lies, then the edge semientire cutvertex graph $e_{c}(G)$ has $(q+c+r)$ vertices and $\sum\left[\frac{d_{i}^{2}}{2}+l_{i}\right]+e_{k}+\frac{c_{i}\left(c_{i}-1\right)}{2}$ edges.

Proof. 3.1: By definition of $e_{c}(G)$, the number of vertices is the sum of edges, cutvertices and regions of $G$. Hence $e_{c}(G)$ has $(q+c+r)$ vertices. Further the number of edges in $n(G)$ is the sum of number of edges incident with cutvertices in $\mathrm{G}$, the number of edges in $\mathrm{L}(\mathrm{G})$. Since the number of edges in $\mathrm{L}(\mathrm{G})$ has $-\mathrm{q}+\sum$ $\frac{d_{i}{ }^{2}}{2}$ and the number of edges in a cutvertex graph is $\frac{c_{i}\left(c_{i}-1\right)}{2}$, hence the number of edges in $e_{c}(G)$ is the sum of edges in $n(G)$ and the number of edges bounded by the regions is $e_{k}$. In addition, the total number of edges that lie on the region $\sum r_{i}$ of $\mathrm{G}$ is $\mathrm{q}$. Thus the number of edges in $\mathrm{e}_{\mathrm{c}}(\mathrm{G})$ is, 


$$
\begin{aligned}
& \mathrm{E}\left[\mathrm{e}_{\mathrm{c}}(\mathrm{G})\right]=-\mathrm{q}+\sum\left[\frac{d_{i}^{2}}{2}+\mathrm{l}_{\mathrm{i}}\right]+\mathrm{q}+\mathrm{e}_{\mathrm{k}}+\frac{c_{i}\left(c_{i}-1\right)}{2} . \\
& =\sum\left[\frac{d_{i}^{2}}{2}+\mathrm{l}_{\mathrm{i}}\right]+\mathrm{e}_{\mathrm{k}}+\frac{c_{i}\left(c_{i}-1\right)}{2} .
\end{aligned}
$$

A criterion for edge semientire cutvertex graph to be planar is presented in our next theorem.

Theorem 3.2: For any graph $G$, the edge semientire cutvertex graph $e_{c}(G)$ is planar if and only if $G$ is a path.

Proof 3.2: Suppose $e_{c}(G)$ is planar. Assume that $G$ is not a path. If there exists a cutvertex $v$ of degree 3 and edges e, $f, g$ are incident to $v$. The cutvertex along with e, $\mathrm{f}, \mathrm{g}$ form $\left\langle\mathrm{K}_{4}\right\rangle$ as a subgraph and the regionvertex is adjacent to $\mathrm{K}_{4}$ to form $\mathrm{K}_{5}-\mathrm{x}$ as a subgraph. Hence $\mathrm{e}_{\mathrm{c}}(\mathrm{G})$ is non-planar, a contradiction. Thus necessity is proved.

For sufficiency, suppose $G$ is a path $P_{t}$ with $t \geq 1$. For $t=1$, the result is obvious. For $t>1$, by definition, each block of lict graph $n(G)$ is $K_{3}$ and which has (t-1) blocks. In addition, $\mathrm{G}$ has exactly one region, the regionvertex together with block form $(t-1)$ number of $\left\langle K_{4}-x\right\rangle$ sub graphs in $e_{c}(G)$. Also the adjacency in cutvertices does not affect the planarity. Hence $e_{c}(G)$ is planar.

Further we develop the nonouterplanar of $e_{c}(G)$ in the following theorem.

Theorem 3.3: For any planar graph $G$, the edge semientire cutvertex graph $e_{c}(G)$ is always nonouterplanar.

Proof 3.3: We have the following cases:

Case 3.1. Suppose $\mathrm{G}$ has a vertex $v$ of degree 3 and edges e, $\mathrm{f}, \mathrm{g}$ are incident to $\mathrm{v}$. The cutvertex along with e, $\mathrm{f}, \mathrm{g}$ form $\left\langle\mathrm{K}_{4}\right\rangle$ as a subgraph and the regionvertex is adjacent to $K_{4}$ to form $K_{5}-x$ as a subgraph. Hence $e_{c}(G)$ is non outerplanar.

Case 3.2. Suppose $G$ is a path $P_{t}$ with $t \geq 1$. For $t=1$, the result is obvious. For $t$ $>1$, by definition, each block of lict graph $n(G)$ is $K_{3}$ and which has (t-1) blocks. In addition, $\mathrm{G}$ has exactly one region, the regionvertex together with block form ( $\mathrm{t}$ $-1)$ number of $\left\langle K_{4}-x\right\rangle$ sub graphs in $e_{c}(G)$. Clearly the internal edges $e_{j}$ for all $j$ of $G$ becomes the inner vertex numbers and is $i\left[e_{c}(G)\right]>1$. Thus $e_{c}(G)$ is nonouterplanar. 
The next theorem gives the minimally nonouterplanar $e_{c}(G)$.

Theorem 3.4: For any planar graph $G$, the edge semientire cutvertex graph $e_{c}(G)$ is minimally nonouterplanar if and only if $G$ is a path $P_{4}$.

Proof 3.4: Suppose edge semientire cutvertex graph $\mathrm{n}_{\mathrm{e}}(\mathrm{G})$ is minimally nonouterplanar. Assume that $G$ is a graph other than $P_{4}$. By the theorem 10, $e_{c}(G)$ has inner vertex number greater than 1 , which is not minimally non outerplanar, a contradiction. Thus necessity is proved.

For sufficiency, suppose $\mathrm{G}$ is a path $\mathrm{P}_{4}$. By the definition of lict graph, each block is $\mathrm{K}_{3}$ and which has 2 blocks. In addition, $\mathrm{G}$ has exactly one region, the regionvertex together with block form two copies of $\left\langle K_{4}-x\right\rangle$ in $e_{c}(G)$. Clearly the internal edges $e_{j}$ of $G$ becomes the inner vertex numbers and is $i\left[e_{c}(G)\right]=1$. Hence $\mathrm{e}_{\mathrm{c}}(\mathrm{G})$ is minimally nonouterplanar.

In the next Theorem, we characterize the edge semientire cutvertex graph in terms of crossing number one.

Theorem 3.5: The edge semientire cutvertex graph $e_{c}(G)$ has a crossing number one if and only if the following conditions hold:

1. $\operatorname{deg} v \leq 3$, for any vertex $v$ of $G$ and

2. $\quad G$ has unique vertex of degree 3 , which is a cutvertex.

Proof 3.5: Suppose $e_{c}(G)$ has crossing number one. Then it is non planar. By Theorem 9 , deg $\mathrm{v} \geq 3$ for any vertex $\mathrm{v}$ of $\mathrm{G}$. We now consider the following cases:

Case 3.3. Assume $G$ has a cutvertex $u$ of degree 4. Then the edges incident to this cutvertex together with the cutvertex form $\left\langle K_{5}\right\rangle$ as a subgraph in $n(G)$, the edges joined by the cutvertices and the regionvertex is adjacent to at least one vertex of $\left\langle K_{5}\right\rangle$ in $e_{c}(G)$. This gives $C\left[e_{c}(G)\right]>1$, a contradiction.

Case 3.4. Assume $\mathrm{G}$ has at least two vertices of degree 4. Suppose $v_{1}$ and $v_{2}$ are two non cutvertices of degree 4. Then $L(G)$ has at least two crossings, by Theorem 2, C $\left[n_{\mathrm{e}}(\mathrm{G})\right]>1$, a contradiction. Suppose $\mathrm{v}_{1}$ and $\mathrm{v}_{2}$ are two cutvertices of degree 4 . Then cutvertices $\mathrm{v}_{1}$ and $\mathrm{v}_{2}$ together with their corresponding incident four edges to form two $\left\langle K_{6}\right\rangle$ as sub graphs in $n(G)$ and hence in $e_{c}(G)$.Hence $\mathrm{C}\left[\mathrm{e}_{\mathrm{c}}(\mathrm{G})\right]>1$, a contradiction. Thus necessity is proved.

For sufficiency, suppose $G$ holds both the conditions of the Theorem. Let $v_{1}$ be the cutvertex of degree 3 . Then by Theorems 2 and 3, n (G) has crossing number one and hence $e_{c}(G)$ has crossing number one.

In the next theorem we characterize the eulerian $e_{c}(G)$. 
Theorem 3.6: For any planar graph $G$, the edge semientire cutvertex graph $e_{c}(G)$ is eulerian if and only if i) $G$ is a caterpillar with spine $P_{5}$ and non-leaves vertices $u, v, w$ having degree $(3,2,3)$ respectively. $i i)$. $G$ is a graph obtained by $a$ path $P_{3}$ joining the cycles $C_{2 n}$ for all $n$ to the edge vertices of $P_{3}$.

Proof. 3.6: Suppose edge semientire cutvertex graph $e_{c}(G)$ is eulerian. Assume that $\mathrm{G}$ is a tree other than caterpillar as mention above. We have the following cases:

Case 3.5. If $T$ is a path $P_{n}=u_{1}, e_{1}, u_{2}, e_{2}, \ldots x_{n-1}, u_{n}$ then, each block of lict graph $\mathrm{n}(\mathrm{G})$ is $\mathrm{K}_{3}$ and which has (n-1) blocks. In addition, $\mathrm{G}$ has exactly one region, the regionvertex together with block form $(n-1)$ number of $\left\langle K_{4}-x\right\rangle$ sub graphs in $\mathrm{e}_{\mathrm{c}}(\mathrm{G})$. Also the cutvertices are adjacent. Here the vertices $\mathrm{x}_{1}, \mathrm{x}_{2}, \ldots \mathrm{x}_{\mathrm{n}-1}$ are adjacent to exactly five vertices and degree of these vertices are even. Hence, it is noneulerian, a contradiction.

Case 3.6. Suppose $T$ is not a path and it contains odd degree of vertices. By the definition of edge semientire cutvertex graph, $e_{c}(G)$ contains a regionvertex of odd degree, which is noneulerian. Further, suppose $\mathrm{T}$ contains even number of edges. By the definition of edge semientire cutvertex graph, the vertex $\mathrm{x}_{\mathrm{i}}$ of odd degree which was the edge in $G$ of even edgedegree. Hence $e_{c}(G)$ is noneulerian, a contradiction.

Case 3.7. Assume that $G$ is a graph obtained by a path $P_{3}$ joining the cycles $C_{n}$ for all $n$ to the edge vertices of $P_{3}$. By the definition of edge semientire cutvertex graph, the interior regionvertices $r_{i}$ and $r_{j}$ have degree odd, and hence $e_{c}(G)$ is noneulerian.

Case 3.8. Assume that $G$ is a graph obtained by a path $P_{4}$ joining the cycles $C_{2 n}$ for all $\mathrm{n}$. By the definition of edge semientire cutvertex graph, the internal edge $\mathrm{e}_{i}$ of $\mathrm{G}$ becomes a vertex of odd degree which is noneulerian, a contradiction. Thus necessity is proved.

For sufficiency, suppose $\mathrm{G}$ satisfies both conditions. We have the following cases:

Case 3.9. $G$ is a caterpillar with spine $P_{5}$ and non-leaves vertices $u, v, w$ having degree $(3,2,3)$ respectively. Clearly, graph $\mathrm{G}$ contains eight edges. Each pendent edge $e_{i}$ is adjacent to two edges and incident to a cutvertex. By the definition of edge semientire cutvertex graph, the vertices $e_{i}$ have degree even. Hence $e_{c}(G)$ is eulerian.

Case 3.10. Suppose $G$ is a graph obtained by a path $P_{3}$ joining the cycles $C_{2 n}$ for all $n$ to the edge vertices of $\mathrm{P}_{3}$. By the definition of lict graph, each vertex is of odd degree. By the definition of edge semientire cutvertex graph, both inner regionvertices have degree even and the exterior regionvertex is also even. Hence $\mathrm{e}_{\mathrm{c}}(\mathrm{G})$ is eulerian.

In the next theorem we characterize the hamiltonian $\mathrm{e}_{\mathrm{c}}(\mathrm{G})$. 
Theorem 3.7: For any planar graph $G$, the edge semientire cutvertex graph $e_{c}(G)$ is always hamiltonian.

Proof. 3.7: Suppose G is any graph. We have the following cases:

Case 3.11.Let $G$ is a path and has exactly one regionvertex. Let $V[n(G)]=\left(e_{1}\right.$, $\left.e_{2}, \ldots e_{n}\right) Y\left(c_{1}, c_{2}, \ldots c_{n-2}\right)$ where $\left(c_{1}, c_{2}, \ldots c_{n-2}\right)$ are cutvertices of G. Since each block is a triangle and each block consist vertices as $B_{1}=\left(e_{1} c_{1} e_{2}\right)$, $B_{2}=\left(e_{2} c_{2} e_{3}\right), \ldots B_{n}=\left(e_{n-1} c_{n-2} e_{n}\right)$.In $n_{e}(G)$, the regionvertex $w$ is adjacent to $\left(e_{1}\right.$, $\left.e_{2}, \ldots e_{n}\right)$. Hence $V\left[e_{c}(G)\right]=\left(e_{1}, e_{2}, \ldots e_{n}\right) Y\left(c_{1}, c_{2}, \ldots c_{n-2}\right) Y w$ form a cycle as $w$

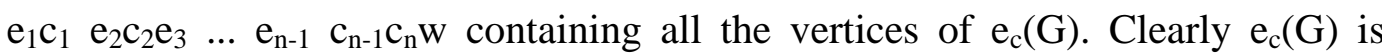
hamiltonian.

Case 3.12.Let $G$ is a tree and has exactly one regionvertex. Let $[n(G)]=\left(e_{1}, e_{2}, \ldots\right.$ $\left.e_{n}\right) Y\left(c_{1}, c_{2}, \ldots c_{j}\right)$ where $\left(c_{1}, c_{2}, \ldots c_{j}\right)$ are the cutvertices of G. Clearly, each block is $\mathrm{K}_{3}$ if degree of the cutvertex is two and is $\mathrm{K}_{4}$ if degree of the cutvertex is three. In $\mathrm{e}_{\mathrm{c}}(\mathrm{G})$, the regionvertex $\mathrm{w}$ is adjacent to $\left(\mathrm{c}_{1}, \mathrm{c}_{2}, \ldots \mathrm{c}_{\mathrm{j}}\right)$. By the remark 2, $e_{c}(G)$ is nonseparable. Clearly, the vertices $\left(e_{1}, e_{2}, \ldots e_{n}\right) Y\left(c_{1}, c_{2}, \ldots c_{j}\right) Y w$ form $w e_{1} c_{1} e_{2} c_{2} e_{3} e_{4} \ldots c_{j} e_{n} w$ containing all the vertices of $e_{c}(G)$. Hence $e_{c}(G)$ is hamiltonian.

Case 3.13 If $\mathrm{G}$ is the graph other than above types of graphs, then by Remark 2, $e_{c}(G)$ is nonseparable, hence $e_{c}(G)$ is hamiltonian.

Case 3.14. If $G$ is hamiltonian graph, then by theorem $7, e_{e}(G)$ is hamiltonian and hence $\mathrm{e}_{\mathrm{c}}(\mathrm{G})$ is hamiltonian.

\section{References}

[1] Harrary, F. Annals of New York Academy of Science, (1977) 175: 198.

[2] Harrary, F. Graph Theory, Addison- Wesley Reading Mass, (1969) , p.72, 107.

[3] Kulli. V. R. and Akka. D. G., Journal mathematical Science, (1980), Vol.14, No. 6, P. 585- 588.

[4] V.R.Kulli and M.H. Muddebihal, Lict Graph and Litact Graph of a Graph, Journal of Analysis and Computation, .(2006),Vol. 2.No. 133-43.

[5] V R Kulli ., On minimally nonouterplanar graphs, Proceeding of the Indian National Science Academy, (1975), Vol 41, part A, No. 3 ,pp 275 - 280.

[6] Sedlacek. J.,Proceeding of the Symposium smolenics, Academic Press, New York, (1963), P. 145.

[7] Y B Maralabhavi and Venkanagouda M Goudar., Lict edge semientire graph of a planar graph., in the journal of Discrete Mathematical Sciences and Cryptography, (2007), vol 10, pp. $433-438$. 